\title{
Evaluaciones internacionales a gran escala y ciudadanía. Explorando la relación entre políticas y ICCS en Chile (2009-2016)
}

\author{
Cristóbal Villalobos ${ }^{1}$ (D) ; Ignacio Wyman ${ }^{1}$ (D) ; Ernesto Treviño ${ }^{1}$ \\ ${ }^{1}$ Pontificia Universidad Católica de Chile (UC), Chile
}

Resumen. El artículo analiza cuatro atributos de la formación ciudadana de los estudiantes (conocimiento cívico, disposición al voto, confianza en los partidos políticos y disposición a compartir con personas de otras clases sociales) considerando la implementación de tres políticas desarrolladas en Chile: i) la Ley de Votof Voluntario (2012); ii) la Ley de Subvención Escolar Preferencial (2008) y; iii) los cambios curriculares a los Planes de Formación Cívica y Ciudadana (2011). Usando los datos de la evaluación ICCS de 2009 ( N=5.192 estudiantes; 177 escuelas) y 2016 (5.081 estudiantes; 178 escuelas) y a través de análisis inferenciales y multinivel, los resultados muestran que existen pocas transformaciones en los promedios entre ambos años, aunque se evidencian cambios cuando se desagregan los datos entre niveles socioeconómicos. Estos resultados permiten reinterpretar la relación entre los ILSA y las políticas educativas, de manera que permitan pensar cómo las Evaluaciones Internacionales a Gran Escala podrían aportar al desarrollo de sistemas educativos para potenciar la ciudadanía en los estudiantes.

Palabras claves: evaluaciones internacionales a gran escala; ciudadanía; desigualdades, ICCS; Chile; política.

\begin{abstract}
Avaliações internacionais em larga escala e cidadania. Explorando a relação entre políticas e o ICCS no Chile (2009-2016)

Resumo. O artigo analisa quatro atributos da formação cidadã dos estudantes (conhecimento cívico, disposição para o voto, confiança nos partidos políticos e disposição para compartilhar com pessoas de outras classes sociais), considerando a implementação de três políticas desenvolvidas no Chile: i) a Lei de Voto Voluntário (2012); ii) a Lei de Subsidio Escolar Preferencial (2008) e; iii) as mudanças curriculares nos Planos de Formação Cívica e Cidadã (2011). Usando os dados da avaliação ICCS de 2009 ( $N=5.192$ estudantes; 177 escolas) e 2016 (5.081 estudantes; 178 escolas) e através de análises estatísticas inferenciais e multinível, os resultados mostram que existem poucas alterações nas médias entre os dois anos, embora evidenciem mudanças ao desagregar os dados entre níveis socioeconômicos. Estes resultados permitem reinterpretar a relação entre os ILSA e as políticas educacionais, permitindo pensar como as Avaliações Internacionais em Larga Escala podem contribuir para o desenvolvimento de sistemas educativos para promover a cidadania nos estudantes.
\end{abstract}

Palavras-chave: avaliações internacionais em larga escala; cidadania; desigualdades, ICCS; Chile; política.

International large-scale assessment and citizenship. Exploring the relationship between politics and ICSS in Chile (2009-2016)

Abstract: This article analyzes four attributes of student citizenship training (civic knowledge, willingness to participate in elections, trust in political parties and willingness to share with people from other social classes) considering the implementation of three policies developed in Chile in recent years: i) the Voluntary Voting Act (2012); ii) the Preferential School Subsidy Act (2008) and; iii) curricular changes in Civic and Citizenship (2011). Using data from Chile from ICCS study in 2009 ( $N=$ 5,192 students; 177 schools) and 2016 (5,081 students; 178 schools), and through inferential and multilevel analyzes, the results show that there are few changes in national averages between both years, although changes are evident when the data is disaggregated by socioeconomic level. These results allow us to re-interpret the relationship between ILSAs and educational policies, to think about how International Large-Scale Assessments may contribute to the development of educational systems that empower students for citizenship.

Keywords: International Large-Scale Assessment; Citizenship; ICCS; Inequalities, Chile; Politics. 


\section{Introducción}

En las últimas décadas, las Evaluaciones Internacionales a Gran Escala o International Large-Scale Assessments (ILSA) han adquirido una creciente importancia en los sistemas educativos del mundo. Este crecimiento puede relacionarse con tres fenómenos convergentes. En primer término, las ILSA se han promovido y potenciado por instituciones supraestatales (organismos internacionales y empresas multinacionales), que buscan difundir, coordinar y promover; pero también restringir, coaccionar o limitar políticas gubernamentales, a través de los llamados procesos de "gobernanza suave" (Bieber, 2016; Niemann y Martens, 2018). En segundo lugar, las ILSA se han expandido a través de la relevancia de lo que Ozga (2008) ha llamado el gobierno del conocimiento, esto es, la tendencia de parte importante de los países a organizar sus políticas a través de procesos de producción y utilización de datos (Grek, 2009), promoviendo procesos de competencia, emulación y/o aprendizaje entre los países (Parcerisa, Fontdevila, y Verger, 2020). Finalmente, las ILSA se han consolidado debido al consenso (hegemónico) sobre la relevancia de la evaluación educativa como un pilar central del proceso escolar (Gorur, 2017; Wagemaker, 2013), que ha promovido no solo la legitimidad sino también la necesidad de estos instrumentos.

Uno de los resultados más llamativos de estos procesos ha sido la multiplicación en intensidad y alcance de estas evaluaciones. Así, en las últimas décadas, las ILSA han incorporado progresivamente a países con menores niveles de desarrollo social (por ejemplo, PISA-D, incorpora a Camboya, Ecuador, Guatemala, Honduras, Panamá, Paraguay, Senegal y Zambia) y a regiones con menos información hasta comienzos del siglo, como Latinoamérica y África (LLECE, SACMEQ y PASEC). En términos de las áreas, las ILSA han involucrado tópicos educativos tan diversos como matemáticas, ciencias, lectura, escritura, habilidades computacionales, competencias digitales, educación financiera y educación cívica y ciudadanía, levantando gran cantidad de información sobre niños, niñas y jóvenes, pero también sobre docentes (en formación y en ejercicio), directores y población adulta.

Este crecimiento explosivo ha motivado una fértil discusión académica sobre los impactos de las ILSA en la política educativa, el currículum, los procesos de enseñanza y las capacidades de medición en distintos países. Aunque se está lejos de alcanzar un consenso sobre los aportes, dificultades y tensiones que generan estos mecanismos, lo cierto es que gran parte de los actores han destacado que las ILSA serían un agente activo de cambio de las políticas (Heyneman y Lee, 2014; Wagemaker, 2013), especial (pero no exclusivamente) en áreas como matemáticas y lectura, que son las áreas más sensibles para la rendición de cuentas (también llamado accountability) de los sistemas educativos. En estos casos, se ha mostrado que Ios ILSA son 
entendidos como una fuente resumida y comparada de la calidad general del sistema escolar, siendo interpretada como una medida del nivel y prestigio educativo de los países (Carnoy, Khavenson e Ivanova, 2013; Sellar, Lingard, Rutkowski y Takayama, 2018).

Sin embargo, la relación entre las políticas gubernamentales y las ILSA que no están directamente relacionados con el accountability escolar ha sido mucho menos explorada, surgiendo distintas interrogantes a este respecto. ¿Qué influencia pueden tener las ILSA en ámbitos no centrales de la rendición de cuentas escolar? ¿Cuál es el uso que los gobiernos hacen de las ILSA no relacionados con el accountability escolar? ¿Cómo, y en qué medida, la implementación de políticas nacionales ha influido en los resultados de evaluaciones internacionales sobre formación cívica y ciudadanía juvenil? Para aproximarnos a estos interrogantes, este artículo se focaliza en una evaluación internacional particular, el International Civic and Citizenship Education Study (ICCS) en un país, Chile. Así, el estudio explora los resultados de ICCS en 2009 y 2016 en cuatro variables de la formación cívica y ciudadana de los estudiantes (conocimiento cívico, disposición al voto, confianza en los partidos políticos y disposición a compartir con personas de otras clases sociales) considerando la implementación de tres políticas que podrían haber impactado en estas mediciones: i) la Ley de Voto Voluntario (2012); ii) la Ley de Subvención Escolar Preferencial (2008) y; iii) los cambios curriculares a los Planes de Formación Cívica y Ciudadana (2011). Particularmente, el estudio tiene tres objetivos específicos: i) comparar los resultados promedio entre 2009 y 2016 en las cuatro variables mencionadas; ii) desagregar los resultados de estas variables en términos socioeconómicos y; iii) analizar la variación del efecto del nivel socioeconómico, pero considerando la influencia de una serie de otras variables, entre los dos años.

Para dar cuenta de estos objetivos, el artículo se estructura en seis apartados además de esta introducción. El segundo apartado describe el ICCS y el impacto de esta prueba en los sistemas educativos. El tercero, se focaliza en describir las características del sistema chileno, con un énfasis en el rol de las ILSA de educación cívica y ciudadanía. El cuarto apartado describe las políticas seleccionadas, argumentando cómo podrían relacionarse con las mediciones de la ICCS. En la quinta sección se describen las variables utilizadas, el método de estimación y análisis realizados. La sexta sección expone los resultados, focalizándose en el cambio de las variables, su distribución y el peso de distintos factores en el tiempo. Finalmente, la séptima sección desliza las principales conclusiones, aportes y limitaciones del estudio. 


\section{El ICCS y su impacto en el desarrollo de políticas educativas}

Administrado por la International Association for the Evaluation of Educational Achievement (IEA), el ICCS puede ser considerado como la ILSA de mayor alcance sobre formación cívica y ciudadana en el mundo ${ }^{1}$. Implementada originalmente en 2009 , el estudio vuelve a aplicarse en 2016 y se espera una tercera ronda para $2022^{2}$, el ICCS recolecta información sobre estudiantes de 13,5 años en promedio. El instrumento recoge información sobre conocimiento, actitudes y acciones de los estudiantes sobre la ciudadanía y la vida cívica (Schulz, Fraillon, Ainley, Losito y Kerr, 2008). Además, incluye información sobre el desarrollo de la educación cívica y ciudadana en la escuela, las experiencias, clima escolar, habilidades y prácticas docentes.

Al contrario de lo que ocurre con otras pruebas como PISA, TALIS o TIMSS, el análisis de la relación entre ICCS y la implementación de políticas educativas y sociales es un ámbito de análisis poco explorado. Las investigaciones existentes al respecto han señalado que el ICCS y el CIVED ha permitido entender y gestionar los procesos de desafección de la identidad nacional, así como los problemas de identidad cultural y étnica de los países (Wagemaker, 2011). Asimismo, se ha destacado que los resultados de las ILSA de educación cívica y ciudadanía han motivado la priorización educativa sobre temas cívicos (por ejemplo, a través de la creación de un curso específico de educación cívica y ciudadana) en ex países comunistas como Estonia, Lituania, Polonia y Eslovenia (Klemencic, 2010). Finalmente, y en términos curriculares, los resultados de ICCS y CIVED motivaron cambios o nuevos planes de estudio en Eslovaquia y Lituania (Pelgrum, Blahova, Dukynaité, Pavešić y Kangro, 2011) pero también influyeron en otros contextos, como Australia (Wagemaker, 2011) y México (Cox, en prensa).

\section{El sistema escolar chileno. Marketización, accountability e ILSA}

Dentro del concierto internacional, el sistema escolar chileno es considerado como un caso extremo de marketización, privatización y consolidación de un sistema universal de vouchers (Villalobos y Quaresma, 2015) Una de sus características más sobresalientes de este sistema es la existencia

${ }^{1}$ En 2009 participaron 35 países, mientras que en 2016 fueron 24. Para la ronda de 2022 participarán 25 países. En todas ellas, el instrumento incluye mayoritariamente países de Europa, y algunos países de América Latina y Asia.

2 En 1971 y 1999, la misma IEA administró la prueba CIVED (Civic Education Study), que también versó sobre aspectos relacionados con la ciudadanía, la democracia, la identidad nacional y la cohesión social, y que puede considerarse como antecedente directo del ICCS. 
de un sistema de rendición de cuentas ligado a pruebas estandarizadas, que premia y castiga a las escuelas según sus resultados (Parcerisa y Falabella, 2017). Por ello, no extraña que Chile sea un terreno fértil para el desarrollo de las ILSA. Actualmente, Chile participa de siete ILSAs: PISA, ICILS, PIRS, TIMMS, ERCE, ECES y ICCS.

La relación entre las ILSA y las políticas educativas en Chile ha sido recientemente analizada. Así, por ejemplo, se ha evidenciado como los resultados de PISA han sido utilizados tanto para entender y describir los "avances" y "estancamientos" del sistema escolar durante las tres primeras décadas desde el retorno a la democracia (Cox y Meckes, 2016). Adicionalmente, los datos de PISA (y en menor medida de TIMSS) han servido como fundamento para el desarrollo de reformas relevantes, como la Ley General de Educación en 2009 o la Ley de Inclusión Escolar en 2015 (Alarcón, 2020; Parcerisa y Verger, 2019). Finalmente, Ios datos de PISA han sido utilizados para discutir sobre "sociedades de referencia", esto es, debatir en torno a los cambios que el sistema educativo chileno debiera desarrollar, teniendo como puntos de referencia países con mejor desempeño, como Finlandia (Parcerisa y Verger, 2019) u otros con modelos de gobernanza semejantes al chileno, como Holanda (Alarcón, 2020).

Respecto de la relación entre las ILSA de educación cívica y ciudadanía y las políticas educativas chilenas, la literatura ha identificado tres impactos. Por una parte, se ha indicado que los marcos de las pruebas internacionales (CIVED e ICCS) han sido una herramienta para apoyar cambios curriculares sobre este tópico en el país durante la década del 2000 (Cariola, Covacevich, Gubler, Lagos y Ortiz, 2011; Cox, en prensa). En segundo término, la existencia de estas pruebas ha fomentado una explotación gubernamental de los datos, permitiendo mejorar la comprensión sobre la materia, desarrollar comisiones de expertos (la Comisión Formación Ciudadana en el 2004) y promover la cooperación internacional (Cariola et al., 2011) sobre estos tópicos. Finalmente, se ha indicado que los resultados de ICCS estarían relacionados con reformas curriculares recientes, como la creación de los Planes de Formación Ciudadana y el Curso de Formación Cívica y Ciudadana para los últimos dos cursos de la secundaria (Cox, en prensa).

Respecto de la relación entre las ILSA de educación cívica y ciudadanía y las políticas educativas chilenas, la literatura ha identificado tres impactos. Por una parte, se ha indicado que los marcos de las pruebas internacionales (CIVED e ICCS) han sido una herramienta para apoyar cambios curriculares sobre este tópico en el país durante la década del 2000 (Cariola, Covacevich, Gubler, Lagos y Ortiz, 2011; Cox, en prensa). En segundo término, la existencia de estas pruebas ha fomentado una explotación gubernamental de los datos, permitiendo mejorar la comprensión sobre la 
materia, desarrollar comisiones de expertos (la Comisión Formación Ciudadana en el 2004) y promover la cooperación internacional (Cariola et al., 2011) sobre estos tópicos. Finalmente, se ha indicado que los resultados de ICCS estarían relacionados con reformas curriculares recientes, como la creación de los Planes de Formación Ciudadana y el Curso de Formación Cívica y Ciudadana para los últimos dos cursos de la secundaria (Cox, en prensa).

\section{Tres políticas en torno a la ciudadanía de la juventud de la última década}

Desde inicios del 2000, en Chile se han desarrollado tres políticas que han buscado transformar el desarrollo cívico y ciudadano de los estudiantes del país. La Tabla 1 describe la política, el ámbito de acción en el que se desenvuelve y las variables del ICCS desde las cuales se podría analizar este cambio, dando cuenta así del marco de análisis desde el que se organiza este artículo.

Tabla 1. Ámbito de acción, política y variables ICCS (2009-2016)

Ámbito Política Variable ICCS

Confianza en los partidos políticos

Actitudes ciudadanas hacia la Ley de Subvención Escolar Disposición a convivir con convivencia con otros Preferencial (2008) otros de distinto nivel socioeconómico

Conocimiento cívico y Ciu- Reformas Curriculares (2009 Conocimiento cívico dadano y 2012)

Fuente: Elaboración propia.

En primer lugar, la Ley de Voto Voluntario (2012) buscó enfrentar el creciente proceso de abstención electoral de la juventud (Carlin, 2006) y la creciente desconfianza en los partidos políticos (INJUV, 2009). Para ello, la Ley transformó el sistema de inscripción desde un registro voluntario y voto obligatorio a uno de registro automático y voto voluntario, derribando así las supuestas "barreras de acceso" para la participación juvenil. Aunque estudios empíricos han mostrado que este cambio no ha generado transformaciones en los patrones de votación de estas nuevas generaciones (Corvalán y Cox, 2013), no se ha explorado suficientemente cómo esta política podría haber afectado las actitudes hacia la institucionalidad política de los jóvenes que aún no tienen el derecho legal a ejercer el voto, como sí permite el ICCS en sus mediciones 2009 y 2016. 
En segundo lugar, y considerando los altos niveles de segregación y polarización del sistema educativo (Valenzuela, Bellei y de los Ríos, 2014) la Ley de Subvención Escolar Preferencial, SEP (2008) entregó más recursos a las escuelas de acuerdo a la vulnerabilidad de sus estudiantes, bajo la condición de eliminar las barreras de ingreso existentes (Weinstein y Villalobos, 2016) ${ }^{3}$. Aunque la evidencia ha mostrado que esta ley ha mejorado los niveles de heterogeneidad sólo en los grupos medios-bajos (Valenzuela, Villaroel y Villalobos, 2013), no se ha explorado mayormente cómo estos cambios podrían estar motivando transformaciones en las actitudes de los jóvenes hacia los otros, lo que es posible realizar gracias a los datos del ICCS.

Finalmente, en el ámbito curricular, y tras importantes cambios que buscaron anclar el currículum escolar hacia ámbitos como la institucionalidad política (Cox y García, 2017), durante 2009 y 2012 se realizaron una serie de modificaciones al currículum en el área de educación cívica y ciudadanía (MINEDUC, 2013). En ambos años, se buscó reforzar la asignatura de Ciencias Sociales e Historia, alineando los contenidos curriculares a los desafíos evidenciados por CIVED 1999 en aspectos como los riesgos de la democracia y los nuevos desafíos de la ciudadanía (Cox, en prensa). En este sentido, estas transformaciones podrían haber promovido cambios en los resultados de conocimiento cívico y en la capacidad de entender los problemas cívicos y ciudadanos de los estudiantes, lo que es medido a través del conocimiento cívico en las pruebas ICCS de 2009 y 2016.

\section{Metodología}

\subsection{Datos}

En este estudio utilizamos los datos para Chile de las versiones de ICCS 2009 y 2016. ICCS es un estudio internacional que recoge información sobre conocimiento y entendimiento de conceptos relacionados con la ciudadanía, así como con las creencias, actitudes y comportamientos de los estudiantes (Schulz, Fraillon, Ainley, Losito y Kerr, 2008). Además, recoge información contextual sobre la enseñanza, organización y prácticas escolares relacionadas con la educación cívica y ciudadana en la escuela. El cuestionario lo compone un módulo general de preguntas y otro específico para las regiones de los países participantes. La recolección de datos ocurre en dos etapas, dentro de cada país. En la primera, se selecciona una muestra representativa de las escuelas con una probabilidad basada en el tamaño de su matrícula.

${ }^{3}$ Estas prohibiciones se extendieron para el conjunto del sistema en el 2015, con la aprobación de la Ley de Inclusión Escolar (LIE). 
En un segundo momento, dentro de cada escuela se selecciona al azar una clase del grado de interés (octavo grado, en el caso chileno). El cuestionario es respondido por estudiantes, directores de escuela y una muestra fija de docentes seleccionados azarosamente entre aquellos que imparten clases al grado de interés. Para el caso chileno, la muestra se compone en 2009 de 5.192 estudiantes pertenecientes a 177 establecimientos, mientras que en 2016 se compone por 5.081 estudiantes de 178 escuelas.

\subsection{Variables de interés}

a) Variables dependientes: La investigación se focaliza en cuatro variables de interés. Por una parte, y relacionado con la implementación de la Ley del Voto Voluntario, se utilizan dos variables: i) la confianza en partidos políticos, que señala el grado de confianza declarado por los estudiantes en estas organizaciones, con un rango de respuesta que va de 0 (no confía) a 3 (confía completamente) y; ii) la participación electoral esperada, que es un índice normalizado para cada país (media 50 y desviación estándar 10 dentro de cada país) creado a partir de tres indicadores: la disposición a votar en futuras elecciones locales, la disposición a votar en elecciones nacionales, y la disposición a informarse sobre los candidatos antes de participar de una elección, donde mayores valores indican una disposición más alta de participación. La tercera variable es la disposición a compartir con otros de distinta clase social, que indica el porcentaje de estudiantes que señalan no tener inconvenientes con compartir en un mismo barrio con personas de distinta clase social ${ }^{4}$, y se utiliza considerando los objetivos de la Ley de Subvención Escolar Preferencial (SEP). Finalmente, para estudiar el posible cambio de las modificaciones curriculares en las áreas de formación cívica y ciudadana, se utiliza el conocimiento cívico, que se mide a través de 87 ítems y se reporta a través de cinco valores plausibles con media 500 y desviación estándar 100 (ambos valores considerando el total de países participantes en el estudio). Puntajes más altos en el indicador muestran mayor conocimiento cívico de los estudiantes.

4 Este indicador se construye siguiendo un enfoque de "análisis de datos integrados" (Rioux y Little, 2020), el cual avala la reconstrucción de indicadores que, difiriendo en la forma exacta de medición entre años, presenta información superpuesta. Así, para el año 2009 el estudiante debía responder entre las siguientes alternativas: "me gustaría", "no me importaría" y "me disgustaría". En el 2016, en cambio: "sí me molesta" y "no me molesta". Para identificar la disposición a compartir con otros de distinta clase social entre estudiantes encuestados el 2009 se considera las alternativas "me gustaría" y "no me molestaría", y "no me molesta", para 2016. 
b) Variable independiente: Las variables dependientes son analizadas en referencia al nivel socioeconómico del hogar del estudiante. Diversos estudios internacionales han señalado al nivel socioeconómico como uno de los factores más relevantes para explicar el conocimiento y comportamiento cívico de estudiantes (Isac, Maslowski, Creemers y van der Werf, 2014; Treviño, Béjares, Villalobos y Naranjo, 2017). Además, es clave cuando la pregunta -como es este caso- se centra en comprender las relaciones entre políticas y evaluaciones de gran escala. Como señalan algunos autores, la atención a las medias nacionales en resultados educativos puede conducir a inferencias erradas sobre los efectos que tienen distintas políticas (TorneyPurta y Amadeo, 2013). Por lo mismo, desagregar estos resultados entre grupos sociales es clave, pues de lo contrario no se estaría reconociendo el altamente probable efecto diferenciado que muchas políticas (si no todas) tienen en sub-grupos poblacionales (Carnoy et al., 2013). En el caso del ICCS, el nivel socioeconómico se construye como un índice a partir de información del estatus ocupacional, el nivel educativo de los padres, y el número de libros en el hogar. Para diferenciar claramente grupos que puedan presentar escenarios disimiles, para esta investigación se decidió trabajar con quintiles de esta variable.

c) Variables de control: Estas variables representan un conjunto de características relacionadas a los individuos, al contexto y a los procesos educativos (Torney-Purta y Amadeo, 2013) que han sido anteriormente incluidas en modelos teórico-conceptuales (Knowles, Torney-Purta y Barber, 2018) y empíricos sobre efectividad escolar en el contexto de la educación cívica (Isac et al., 2014; Treviño et al., 2017; Treviño, Villalobos, Béjares y Naranjo, 2019). Así, se incluyen variables críticas a nivel estudiante (género, el interés de los padres en política y la frecuencia con que los jóvenes discuten asuntos políticos fuera de la escuela) y de la escuela (nivel socioeconómico y dependencia pública o privada) y variables relacionadas con procesos educativos (apertura de la clase para la discusión), que han sido señaladas repetidamente como factores críticos para evaluar distintas variables cívicas (Knowles et al., 2018)

\subsection{Estrategia de análisis}

El análisis se desarrolla a través de un proceso organizado en tres fases. En primer lugar, y a través de análisis inferencial, se discuten los cambios en el tiempo de las medias de las cuatro variables en 2009 y 2016. En un segundo momento, las medias se desagregan según el nivel socioeconómico de los estudiantes. De esta forma, se busca explorar posibles diferencias asociadas al capital socioeconómico de los jóvenes en las distintas variables estudiadas, buscando entender el posible efecto diferenciado de la 
política según posición social (Carnoy et al., 2013). Finalmente, la tercera fase presenta modelos multinivel ${ }^{5}$, considerando la estructura de los datos de la prueba (estudiantes anidados a escuelas) que buscan analizar -para cada variable y para cada año- la variación del efecto del nivel socioeconómico, controlando por una serie de variables críticas tanto a nivel de estudiante como de escuela. De esta forma, se busca explorar si la magnitud del efecto podría estar cambiando entre 2009 y 2016 en los cuatro indicadores analizados. Para cada variable dependiente se especifican dos modelos por año. En su primera especificación sólo se incluye como predictor el nivel socioeconómico y el género del estudiante. En la segunda, se incluyen otras variables de control a nivel del estudiante y de la escuela. En todos los casos, los análisis se realizan usando el software $\mathrm{R}$, usando los pesos muestrales correspondientes.

\section{Resultados}

La Tabla 2 presenta la media de las cuatro variables dependientes para 2009 y 2016 . Como se puede observar, en dos variables (conocimiento cívico y disposición a participar en elecciones) no se observan diferencias estadísticamente significativas entre 2009 y 2016. Respecto del conocimiento cívico, esto podría tener relación con que la implementación de reformas curriculares no es automática, sino que implica procesos de apropiación por parte de los docentes, que deben re-significar los cambios curriculares en sus propias prácticas (Ball, 2003). En el caso de la disposición al voto, la mantención de la disposición está en línea con la actuación que los patrones de votación que han mostrado los jóvenes, que se han mantenido inalterados a pesar del cambio en la legislación (Corvalán y Cox, 2013).

${ }^{5}$ Los modelos multinivel permiten analizar el impacto de una o un conjunto de covariables en una variable dependiente considerando el anidamiento de estudiantes en escuelas (Hox, Moerbeek y Van de Schoot, 2017). De esta manera, se controla el efecto de pertenencia de un grupo de unidades a una de mayor escala, lo que en caso de omisión, podría llevar a inferencias incorrectas (error tipo 1). La especificación general del modelo es: $Y i_{j k}=\beta_{0 j}+\beta_{1 j} X_{i j}+r_{i j}(1)$

$\beta_{0 j}=\gamma_{00}+\gamma_{01} W_{j}+v_{0 j}(2)$ $\beta_{1 j}^{0 j}=Y_{01}$ (3) donde $\mathrm{Y}$ es la variable dependiente y $\mathrm{X}$ el conjunto de covariables a nivel de estudiante (1). En (2), W representa las variables a nivel de escuela, mientras que (3) señala que los modelos estimados consideran pendientes fijas. 
Tabla 2. Diferencias de las medias entre 2009 y 2016

\begin{tabular}{lcrrr}
\hline \multicolumn{1}{c}{ Variable } & $\begin{array}{c}\text { Año } \\
\text { medición }\end{array}$ & Media & \multicolumn{1}{c}{$\begin{array}{c}\text { Límite } \\
\text { superior }\end{array}$} & \multicolumn{1}{c}{$\begin{array}{c}\text { Límite } \\
\text { inferior }\end{array}$} \\
Conocimiento Cívico & 2009 & 483,02 & 485,41 & 480,64 \\
& 2016 & 482,45 & 485,06 & 479,85 \\
Disposición a participar en elec- & 2009 & 49,83 & 50,17 & 49,49 \\
ciones & 2016 & 50,17 & 50,46 & 49,89 \\
Confianza en partidos políticos & 2009 & $2,80 *$ & 2,82 & 2,78 \\
& 2016 & $2,86 *$ & 2,88 & 2,83 \\
Disposición a compartir con perso- & 2009 & $95,05 \% *$ & $95,64 \%$ & $94,46 \%$ \\
nas de distinta clase social & 2016 & $90,41 \% *$ & $91,23 \%$ & $89,60 \%$ \\
\hline
\end{tabular}

Nota: ${ }^{*} p<.05$. Límite inferior y Límite superior de los intervalos al 95\% de confianza ${ }^{6}$. Fuente: elaboración propia.

Por otra parte, se evidencia un aumento estadísticamente significativo (pero mínimo) en el nivel de confianza con los partidos políticos. Este aumento llama la atención, ya que distintos autores han mostrado la baja legitimidad de estas instituciones. Sin embargo, es posible hipotetizar que este aumento podría deberse a los cambios en los clivajes políticos experimentados en la última década que han generado el surgimiento de nuevas fuerzas y una mayor presencia de figuras jóvenes en la política nacional (Alvarado, Rivera y Morales, 2019). Finalmente, los datos revelan que la disposición a compartir con otros de distinta clase social ha disminuido entre 2009 y 2016. Hipotéticamente, podría pensarse que el aumento de la discusión sobre las desigualdades escolares promovidas por la SEP (pero también por otras legislaciones, como la LGE, y por intensos procesos de movilización social en 2006 y 2011) hayan profundizado temores subterráneos, disminuyendo la propensión a compartir con "otros", discurso especialmente presente en los grupos medios (Canales, Bellei y Orellana, 2016).

Para indagar en las diferencias según nivel socioeconómico, la Tabla 3 describe las medias y límites (inferiores y superiores) para cada quintil obtenidos mediante regresiones con errores estándares robustos, mostrando importantes variaciones "escondidas" en la media poblacional.

6 Cuando ambos límites se encuentran en el mismo cuadrante (mantienen el mismo signo), los efectos son estadísticamente significativos al 95\% de confianza. 
Tabla 3. Medias según quintil.

\begin{tabular}{|c|c|c|c|c|c|c|c|}
\hline \multirow[b]{2}{*}{ Variable } & \multirow[b]{2}{*}{ NSE } & \multicolumn{3}{|c|}{ ICCS 2009} & \multicolumn{3}{|c|}{ ICCS 2016} \\
\hline & & Media & $\begin{array}{l}\text { Límite } \\
\text { superior }\end{array}$ & $\begin{array}{l}\text { Límite } \\
\text { inferior }\end{array}$ & Media & $\begin{array}{l}\text { Límite } \\
\text { superior }\end{array}$ & $\begin{array}{l}\text { Límite } \\
\text { inferior }\end{array}$ \\
\hline \multirow{5}{*}{$\begin{array}{l}\text { Conocimiento } \\
\text { cívico }\end{array}$} & 1 & 442,62 & 453,67 & 431,57 & 435,93 & 444,93 & 426,93 \\
\hline & 2 & 461,80 & 474,58 & 449,02 & 465,23 & 474,81 & 455,65 \\
\hline & 3 & 483,78 & 495,87 & 471,69 & 483,66 & 493,70 & 473,62 \\
\hline & 4 & 510,26 & 522,12 & 498,40 & 510,10 & 521,04 & 499,16 \\
\hline & 5 & 548,36 & 562,32 & 534,40 & 544,07 & 555,93 & 532,21 \\
\hline \multirow{5}{*}{$\begin{array}{l}\text { Disposición a } \\
\text { participar en } \\
\text { elecciones }\end{array}$} & 1 & 49,36 & 50,33 & 48,38 & 47,96 & 48,72 & 47,20 \\
\hline & 2 & 49,03 & 50,25 & 47,81 & 49,08 & 50,08 & 48,08 \\
\hline & 3 & 48,95 & 50,34 & 47,56 & 50,21 & 51,17 & 49,25 \\
\hline & 4 & 49,90 & 51,39 & 48,41 & 51,21 & 52,29 & 50,13 \\
\hline & 5 & 52,98 & 54,41 & 51,55 & 53,70 & 54,64 & 52,76 \\
\hline \multirow{5}{*}{$\begin{array}{l}\text { Confianza en } \\
\text { partidos polí- } \\
\text { ticos }\end{array}$} & 1 & 2,69 & 2,76 & 2,63 & 2,77 & 2,85 & 2,70 \\
\hline & 2 & 2,75 & 2,83 & 2,67 & 2,85 & 2,95 & 2,75 \\
\hline & 3 & 2,85 & 2,95 & 2,75 & 2,87 & 2,95 & 2,79 \\
\hline & 4 & 2,90 & 3.00 & 2,80 & 2,92 & 3,02 & 2,82 \\
\hline & 5 & 2,82 & 2,92 & 2,72 & 2,88 & 3.00 & 2,76 \\
\hline \multirow{5}{*}{$\begin{array}{l}\text { Disposición a } \\
\text { compartir con } \\
\text { personas de } \\
\text { distinta clase } \\
\text { social }\end{array}$} & 1 & $93.3 \% *$ & $95,01 \%$ & $91,58 \%$ & $87.88 \% *$ & $90,17 \%$ & $85,59 \%$ \\
\hline & 2 & $95,60 \%$ & $97,68 \%$ & $93,52 \%$ & $90,85 \%$ & $93,93 \%$ & $87,77 \%$ \\
\hline & 3 & $95,86 \%$ & $97,98 \%$ & $93,74 \%$ & $92,29 \%$ & $95,21 \%$ & $89,37 \%$ \\
\hline & 4 & $95,44 \%$ & $97,67 \%$ & $93,21 \%$ & $92,11 \%$ & $95,01 \%$ & $89,21 \%$ \\
\hline & 5 & $93,80 \%$ & $96,23 \%$ & $91,37 \%$ & $91,67 \%$ & $95,06 \%$ & $88,28 \%$ \\
\hline
\end{tabular}

Nota: ${ }^{*} p<.05$. Límite inferior y Límite superior de los intervalos al 95\% de confianza Fuente: Elaboración propia a partir de datos ICCS 2009 y 2016.

Así, pareciera ser que el cambio en la Ley de Voto Voluntario podría haber afectado de manera distinta la disposición a participar en elecciones en el futuro. La disposición a esta acción cívica habría disminuido en el quintil 1 y aumentado en la clase media y alta (quintiles 3,4 y 5) aunque no de manera estadísticamente significativa. Esto podría estar indicando, tal como han mostrado otros estudios, que la generación de mayores niveles de voluntariedad podría estar afectando las posibilidades de participación política de los jóvenes más vulnerables, que no verían en el voto un acto que pudiera transformar la realidad social (Corvalán y Cox, 2013).

En segundo lugar, la desagregación por quintiles muestra que el aumento promedio en la confianza en los partidos políticos está explicado principalmente por un incremento estadísticamente significativo entre los 
estudiantes del primer quintil. Tal hallazgo es complementario al anterior, ya que se visualiza que, en el quintil más bajo existiría, al mismo tiempo, una disminución de la disposición a votar y un aumento en la confianza en los partidos políticos. Se trata de una compleja combinación que podría incentivar el clientelismo entre los partidos políticos para promover el voto y que nos parece que es necesario explorar con mayor profundidad en el futuro.

Por otra parte, la disposición a compartir con otros de distinta clase social disminuyó en forma generalizada entre los quintiles 1 al 4, y con especial fuerza en el primer quintil. En contraste, en el quintil 5 no hay diferencias significativas en torno a esta variable. Estos resultados podrían estar dando cuenta de la plausibilidad de la hipótesis de "reacción" en un contexto de polarización del sistema escolar (Villalobos y Valenzuela, 2012), ya que es precisamente en el quintil 5 donde los cambios impulsados por la SEP (y luego profundizados por la LIE) no operarían en su totalidad, ya que estas regulaciones no afectan a los establecimientos privados sin subvención estatal que están concentrados en este quintil. En contraste, es en los sectores más vulnerables (quintil 1) donde la política podría haber desarrollado más transformaciones en la convivencia con sectores distintos, lo que en parte podría explicar esta disminución a la disposición a compartir con otros sectores sociales.

Finalmente, y para explorar el peso relativo de los factores asociados a distintos resultados cívicos entre 2009 y 2016 se presentan los resultados de los modelos multinivel. Las Tablas 4 y 5 presentan los resultados de las variables relacionadas con el cambio en la Ley de Voto Voluntario: disposición a participar en las elecciones y confianza en los partidos políticos. La Tabla 4 se focaliza en la disposición a participar en las elecciones. Se puede observar, en primer término, que las variables individuales adquieren significancia en 2016 en los modelos que presentan sólo las variables de nivel socioeconómico, además de género (Modelos 1 y 3). Sin embargo, tanto en 2009 como en 2016 los modelos que controlan por características individuales y de la escuela (Modelos 2 y 4) muestran que el género y nivel socioeconómico no son estadísticamente significativas. En contraste, la mayoría de las variables de control (interés de padres en política, discutir sobre política fuera de la escuela, discusión abierta en la sala de clases) son significativas en ambos años y mantienen magnitudes relativamente iguales. Finalmente, se puede observar que el nivel socioeconómico a nivel de la escuela adquiere significancia sólo en 2016, lo que podría estar indicando que la composición socioeconómica podría ser una variable que haya adquirido mayor relevancia en los últimos años. 
Tabla 4. Modelos multinivel. Disposición a participar en elecciones

\begin{tabular}{|c|c|c|c|c|c|c|c|c|}
\hline & \multicolumn{4}{|c|}{2009} & \multicolumn{4}{|c|}{2016} \\
\hline & \multicolumn{2}{|c|}{ Modelo 1} & \multicolumn{2}{|c|}{ Modelo 2} & \multicolumn{2}{|c|}{ Modelo 3} & \multicolumn{2}{|c|}{ Modelo 4} \\
\hline & $\begin{array}{l}\text { Coef } \\
\text { (SE) }\end{array}$ & LI, LS & $\begin{array}{l}\text { Coef } \\
\text { (SE) }\end{array}$ & LI, LS & $\begin{array}{l}\text { Coef } \\
\text { (SE) }\end{array}$ & LI, LS & $\begin{array}{l}\text { Coef } \\
\text { (SE) }\end{array}$ & LI, LS \\
\hline Intercepto & $\begin{array}{l}49.22^{*} \\
(0.55)\end{array}$ & $\begin{array}{l}{[48.14} \\
50.31]\end{array}$ & $\begin{array}{l}51.03^{*} \\
(0.68)\end{array}$ & $\begin{array}{l}\text { [49.7, } \\
52.36]\end{array}$ & $\begin{array}{l}47.4^{*} \\
(0.42)\end{array}$ & $\begin{array}{l}{[46.59,} \\
48.22]\end{array}$ & $\begin{array}{l}50.53^{*} \\
(0.54)\end{array}$ & $\begin{array}{l}\text { [49.47, } \\
51.58]\end{array}$ \\
\hline $\begin{array}{l}\text { Género (Fe- } \\
\text { menino) }\end{array}$ & $\begin{array}{c}0.33 \\
(0.46)\end{array}$ & $\begin{array}{c}{[-0.56} \\
1.22]\end{array}$ & $\begin{array}{l}-0.25 \\
(0.45)\end{array}$ & $\begin{array}{c}{[-1.12,} \\
0.62]\end{array}$ & $\begin{array}{l}1.11^{*} \\
(0.4)\end{array}$ & $\begin{array}{c}{[0.33} \\
1.89]\end{array}$ & $\begin{array}{c}0.74 \\
(0.38)\end{array}$ & $\begin{array}{c}{[-0.01,} \\
1.49]\end{array}$ \\
\hline $\begin{array}{l}\text { NSE quin- } \\
\text { til } 2\end{array}$ & $\begin{array}{l}-0.39 \\
(0.63)\end{array}$ & $\begin{array}{c}{[-1.64} \\
0.85]\end{array}$ & $\begin{array}{l}-1.09 \\
(0.64)\end{array}$ & $\begin{array}{c}{[-2.34} \\
0.16]\end{array}$ & $\begin{array}{l}1.09 * \\
(0.51)\end{array}$ & $\begin{array}{l}{[0.09} \\
2.08]\end{array}$ & $\begin{array}{c}-0.3 \\
(0.57)\end{array}$ & $\begin{array}{c}{[-1.41} \\
0.81]\end{array}$ \\
\hline $\begin{array}{l}\text { NSE quin- } \\
\text { til } 3\end{array}$ & $\begin{array}{l}-0.4 \\
(0.71)\end{array}$ & $\begin{array}{l}{[-1.8,} \\
0.99]\end{array}$ & $\begin{array}{l}-1.39 \\
(0.65)\end{array}$ & $\begin{array}{l}{[-2.67} \\
-0.11]\end{array}$ & $\begin{array}{l}2.27^{*} \\
(0.49)\end{array}$ & $\begin{array}{l}{[1.31} \\
3.22]\end{array}$ & $\begin{array}{c}0.34 \\
(0.57)\end{array}$ & $\begin{array}{c}{[-0.79} \\
1.46]\end{array}$ \\
\hline $\begin{array}{l}\text { NSE quin- } \\
\text { til } 4\end{array}$ & $\begin{array}{c}0.52 \\
(0.77)\end{array}$ & $\begin{array}{c}{[-0.99} \\
2.02]\end{array}$ & $\begin{array}{l}-1.16 \\
(0.76)\end{array}$ & $\begin{array}{c}{[-2.64} \\
0.32]\end{array}$ & $\begin{array}{l}3.3^{*} \\
(0.55)\end{array}$ & $\begin{array}{l}{[2.23} \\
4.38]\end{array}$ & $\begin{array}{l}-0.23 \\
(0.61)\end{array}$ & $\begin{array}{c}{[-1.43} \\
0.97]\end{array}$ \\
\hline $\begin{array}{l}\text { NSE quin- } \\
\text { til } 5\end{array}$ & $\begin{array}{l}3.59^{*} \\
(0.73)\end{array}$ & $\begin{array}{l}{[2.16,} \\
5.02]\end{array}$ & $\begin{array}{c}0.68 \\
(0.89)\end{array}$ & $\begin{array}{c}{[-1.07} \\
2.43]\end{array}$ & $\begin{array}{l}5.72^{*} \\
(0.47)\end{array}$ & $\begin{array}{l}{[4.8} \\
6.64]\end{array}$ & $\begin{array}{c}0.62 \\
(0.61)\end{array}$ & $\begin{array}{c}{[-0.58,} \\
1.82]\end{array}$ \\
\hline $\begin{array}{l}\text { Interés de } \\
\text { padres en } \\
\text { política }\end{array}$ & & & $\begin{array}{l}2.02^{*} \\
(0.18)\end{array}$ & $\begin{array}{c}{[1.67,} \\
2.36]\end{array}$ & & & $\begin{array}{l}2.14^{*} \\
(0.21)\end{array}$ & $\begin{array}{l}{[1.73,} \\
2.55]\end{array}$ \\
\hline $\begin{array}{l}\text { Discutir so- } \\
\text { bre política } \\
\text { fuera de la } \\
\text { escuela }\end{array}$ & & & $\begin{array}{l}2.28^{*} \\
(0.22)\end{array}$ & $\begin{array}{l}{[1.84} \\
2.71]\end{array}$ & & & $\begin{array}{l}1.67^{*} \\
(0.22)\end{array}$ & $\begin{array}{l}{[1.24,} \\
2.09]\end{array}$ \\
\hline $\begin{array}{l}\text { Dependen- } \\
\text { cia (privado) }\end{array}$ & & & $\begin{array}{c}-0.4 \\
(0.58)\end{array}$ & $\begin{array}{c}{[-1.53} \\
0.74]\end{array}$ & & & $\begin{array}{l}-0.74 \\
(0.4)\end{array}$ & $\begin{array}{c}{[-1.52,} \\
0.04]\end{array}$ \\
\hline $\begin{array}{l}\text { NSE Es- } \\
\text { cuela }\end{array}$ & & & $\begin{array}{l}0.18 \\
(0.4)\end{array}$ & $\begin{array}{l}{[-0.6} \\
0.97]\end{array}$ & & & $\begin{array}{l}1.21^{*} \\
(0.27)\end{array}$ & $\begin{array}{c}{[0.68,} \\
1.73]\end{array}$ \\
\hline $\begin{array}{l}\text { Discusión } \\
\text { abierta en } \\
\text { clases }\end{array}$ & & & $\begin{array}{l}0.94^{*} \\
(0.27)\end{array}$ & $\begin{array}{c}{[0.42} \\
1.47]\end{array}$ & & & $\begin{array}{l}0.85^{*} \\
(0.21)\end{array}$ & $\begin{array}{c}{[0.44,} \\
1.26]\end{array}$ \\
\hline ICC & 0.06 & & 0.05 & & 0.06 & & 0.06 & \\
\hline
\end{tabular}

Nota: ${ }^{*} \mathrm{p}<.05$; LI y LS son los límites inferior y superior de los intervalos al $95 \%$ de confianza. Fuente: elaboración propia

Por otra parte, la Tabla 5, que presenta los resultados para la variable confianza en partidos políticos, muestra que tanto el género como el nivel socioeconómico presentan resultados estadísticamente significativos en ambos años, en todos los casos a favor de las mujeres y de los niveles socioeconómicos más altos, tanto en 2009 como en 2016. Es interesante notar que, en términos socioeconómicos, las mayores brechas están entre el quintil 1 (de referencia) y los quintiles que representan las clases medias y medias-altas (quintiles 3 y 4 ) y que además la magnitud del cambio tiende a disminuir entre 2009 y 2016, aunque de manera no significativa. 
Tabla 5. Modelo multinivel. Confianza en partidos políticos

\begin{tabular}{|c|c|c|c|c|c|c|c|c|}
\hline & \multicolumn{4}{|c|}{2009} & \multicolumn{4}{|c|}{2016} \\
\hline & \multicolumn{2}{|c|}{ Modelo 1} & \multicolumn{2}{|c|}{ Modelo 2} & \multicolumn{2}{|c|}{ Modelo 3} & \multicolumn{2}{|c|}{ Modelo 4} \\
\hline & $\begin{array}{l}\text { Coef } \\
\text { (se) }\end{array}$ & LI, LS & $\begin{array}{l}\text { Coef } \\
\text { (se) }\end{array}$ & LI, LS & $\begin{array}{l}\text { Coef } \\
\text { (se) }\end{array}$ & LI, LS & $\begin{array}{l}\text { Coef } \\
\text { (se) }\end{array}$ & LI, LS \\
\hline Intercepto & $\begin{array}{l}2.66^{*} \\
(0.04)\end{array}$ & $\begin{array}{r}{[2.58} \\
2.73]\end{array}$ & $\begin{array}{l}2.64^{*} \\
(0.04)\end{array}$ & $\begin{array}{l}{[2.57} \\
2.72]\end{array}$ & $\begin{array}{l}2.71^{*} \\
(0.04)\end{array}$ & $\begin{array}{l}{[2.63} \\
2.79]\end{array}$ & $\begin{array}{l}2.68^{*} \\
(0.05)\end{array}$ & $\begin{array}{r}{[2.58} \\
2.78]\end{array}$ \\
\hline $\begin{array}{l}\text { Género (Feme- } \\
\text { nino) }\end{array}$ & $\begin{array}{l}0.07^{*} \\
(0.02)\end{array}$ & $\begin{array}{c}{[0.02} \\
0.11]\end{array}$ & $\begin{array}{l}0.09^{*} \\
(0.02)\end{array}$ & $\begin{array}{l}{[0.05} \\
0.13]\end{array}$ & $\begin{array}{l}0.12^{*} \\
(0.03)\end{array}$ & $\begin{array}{l}{[0.06} \\
0.18]\end{array}$ & $\begin{array}{l}0.14^{*} \\
(0.03)\end{array}$ & $\begin{array}{c}{[0.08,} \\
0.2]\end{array}$ \\
\hline NSE quintil 2 & $\begin{array}{l}0.07 \\
(0.04)\end{array}$ & $\begin{array}{c}{[-0.02} \\
0.15]\end{array}$ & $\begin{array}{c}0.08 \\
(0.04)\end{array}$ & $\begin{array}{c}{[0,} \\
0.16]\end{array}$ & $\begin{array}{l}0.08 \\
(0.05)\end{array}$ & $\begin{array}{c}{[-0.02} \\
0.18]\end{array}$ & $\begin{array}{c}0.04 \\
(0.05)\end{array}$ & $\begin{array}{r}{[-0.06} \\
0.14]\end{array}$ \\
\hline NSE quintil 3 & $\begin{array}{l}0.16^{*} \\
(0.05)\end{array}$ & $\begin{array}{c}{[0.07} \\
0.26]\end{array}$ & $\begin{array}{l}0.18^{*} \\
(0.05)\end{array}$ & $\begin{array}{l}{[0.08} \\
0.28]\end{array}$ & $\begin{array}{l}0.1^{*} \\
(0.05)\end{array}$ & $\begin{array}{l}{[0.01} \\
0.19]\end{array}$ & $\begin{array}{l}0.07 \\
(0.05)\end{array}$ & $\begin{array}{c}{[-0.02} \\
0.17]\end{array}$ \\
\hline NSE quintil 4 & $\begin{array}{l}0.21^{*} \\
(0.05)\end{array}$ & $\begin{array}{l}{[0.12} \\
0.31]\end{array}$ & $\begin{array}{l}0.25^{*} \\
(0.05)\end{array}$ & $\begin{array}{c}{[0.14} \\
0.35]\end{array}$ & $\begin{array}{l}0.16^{*} \\
(0.05)\end{array}$ & $\begin{array}{l}{[0.07} \\
0.25]\end{array}$ & $\begin{array}{l}0.12^{*} \\
(0.06)\end{array}$ & $\begin{array}{r}{[0.01} \\
0.23]\end{array}$ \\
\hline NSE quintil 5 & $\begin{array}{l}0.13^{*} \\
(0.05)\end{array}$ & $\begin{array}{l}{[0.02} \\
0.24]\end{array}$ & $\begin{array}{l}0.19^{*} \\
(0.06)\end{array}$ & $\begin{array}{l}{[0.07} \\
0.31]\end{array}$ & $\begin{array}{c}0.11 \\
(0.06)\end{array}$ & $\begin{array}{c}{[0,} \\
0.23]\end{array}$ & $\begin{array}{c}0.06 \\
(0.07)\end{array}$ & $\begin{array}{c}{[-0.09} \\
0.21]\end{array}$ \\
\hline $\begin{array}{l}\text { Interés de padres } \\
\text { en política }\end{array}$ & & & $\begin{array}{l}-0.14^{*} \\
(0.02)\end{array}$ & $\begin{array}{l}{[-0.17} \\
-0.11]\end{array}$ & & & $\begin{array}{l}-0.16^{*} \\
(0.02)\end{array}$ & $\begin{array}{l}{[-0.19} \\
-0.12]\end{array}$ \\
\hline $\begin{array}{l}\text { Discutir sobre } \\
\text { política fuera de } \\
\text { la escuela }\end{array}$ & & & $\begin{array}{l}-0.07 * \\
(0.01)\end{array}$ & $\begin{array}{l}{[-0.1} \\
-0.04]\end{array}$ & & & $\begin{array}{c}0 \\
(0.02)\end{array}$ & $\begin{array}{c}{[-0.03} \\
0.03]\end{array}$ \\
\hline $\begin{array}{l}\text { Dependencia } \\
\text { (privado) }\end{array}$ & & & $\begin{array}{l}-0.03^{*} \\
(0.05)\end{array}$ & $\begin{array}{c}{[-0.13} \\
0.07]\end{array}$ & & & $\begin{array}{l}0.1^{*} \\
(0.04)\end{array}$ & $\begin{array}{r}{[0.02} \\
0.18]\end{array}$ \\
\hline NSE Escuela & & & $\begin{array}{c}0.05 \\
(0.02)\end{array}$ & $\begin{array}{l}{[0,} \\
0.1]\end{array}$ & & & $\begin{array}{l}0.07^{*} \\
(0.03)\end{array}$ & $\begin{array}{r}{[0.02} \\
0.12]\end{array}$ \\
\hline $\begin{array}{l}\text { Discusión abierta } \\
\text { en clases }\end{array}$ & & & $\begin{array}{l}-0.03 \\
(0.02)\end{array}$ & $\begin{array}{c}{[-0.07} \\
0.01]\end{array}$ & & & $\begin{array}{l}-0.02 \\
(0.02)\end{array}$ & $\begin{array}{r}{[-0.06,} \\
0.01]\end{array}$ \\
\hline ICC & 0.03 & & 0.03 & & 0.03 & & 0.03 & \\
\hline
\end{tabular}

Nota: ${ }^{*} \mathrm{p}<.05$; LI y LS son los límites inferior y superior de los intervalos al 95\% de confianza. Fuente: elaboración propia

La Tabla 6 presenta los resultados sobre disposición a compartir con otros de distinta clase. Como se puede ver, al controlar por otros factores, el peso del nivel socioeconómico es prácticamente la única variable significativa del modelo, tanto para el 2009 como el 2016. En ambos años, la diferencia entre el quintil y los grupos medios (quintiles 2, 3 y 4) es significativa y positiva, lo que podría estar confirmando la idea anterior: que la disposición a compartir con otros sería menor en los quintiles extremos (quintil 1 y 5). Adicionalmente, y de manera diferenciada con otras variables, ninguna de las variables de la escuela ni de los procesos escolares es estadísticamente significativa para los años analizados. 
Tabla 6. Modelo multinivel. Disposición a compartir con otros de distinta clase

\begin{tabular}{|c|c|c|c|c|c|c|c|c|}
\hline & \multicolumn{4}{|c|}{2009} & \multicolumn{4}{|c|}{2016} \\
\hline & \multicolumn{2}{|c|}{ Modelo 1} & \multicolumn{2}{|c|}{ Modelo 2} & \multicolumn{2}{|c|}{ Modelo 3} & \multicolumn{2}{|c|}{ Modelo 4} \\
\hline & $\begin{array}{l}\text { Coef } \\
\text { (se) }\end{array}$ & LI, LS & $\begin{array}{l}\text { Coef } \\
\text { (se) }\end{array}$ & LI, LS & $\begin{array}{l}\text { Coef } \\
\text { (se) }\end{array}$ & LI, LS & $\begin{array}{l}\text { Coef } \\
\text { (se) }\end{array}$ & LI, LS \\
\hline Intercepto & $\begin{array}{l}0.93^{*} \\
(0.01)\end{array}$ & $\begin{array}{l}{[0.91} \\
0.95]\end{array}$ & $\begin{array}{l}0.93^{*} \\
(0.01)\end{array}$ & $\begin{array}{l}{[0.91} \\
0.95]\end{array}$ & $\begin{array}{l}0.86^{*} \\
(0.01)\end{array}$ & $\begin{array}{l}{[0.84} \\
0.89]\end{array}$ & $\begin{array}{l}0.86^{*} \\
(0.02)\end{array}$ & $\begin{array}{l}{[0.83} \\
0.89]\end{array}$ \\
\hline $\begin{array}{l}\text { Género (Feme- } \\
\text { nino) }\end{array}$ & $\begin{array}{l}0.01 \\
(0.01)\end{array}$ & $\begin{array}{l}{[-0.01} \\
0.02]\end{array}$ & $\begin{array}{l}0.01 \\
(0.01)\end{array}$ & $\begin{array}{l}{[-0.01} \\
0.02]\end{array}$ & $\begin{array}{l}0.03^{*} \\
(0.01)\end{array}$ & $\begin{array}{l}{[0.01} \\
0.05]\end{array}$ & $\begin{array}{l}0.03^{*} \\
(0.01)\end{array}$ & $\begin{array}{l}{[0.01} \\
0.05]\end{array}$ \\
\hline NSE quintil 2 & $\begin{array}{l}0.02 \\
(0.01)\end{array}$ & $\begin{array}{l}{[0,} \\
0.04]\end{array}$ & $\begin{array}{l}0.03^{*} \\
(0.01)\end{array}$ & $\begin{array}{l}{[0.01} \\
0.05]\end{array}$ & $\begin{array}{l}0.03 \\
(0.02)\end{array}$ & $\begin{array}{l}{[0,00} \\
0.06]\end{array}$ & $\begin{array}{l}0.03 \\
(0.02)\end{array}$ & $\begin{array}{l}{[0,00} \\
0.06]\end{array}$ \\
\hline NSE quintil 3 & $\begin{array}{l}0.03 \\
(0.01)\end{array}$ & $\begin{array}{l}{[0,} \\
0.05]\end{array}$ & $\begin{array}{l}0.03^{*} \\
(0.01)\end{array}$ & $\begin{array}{l}{[0.01} \\
0.05]\end{array}$ & $\begin{array}{l}0.04^{*} \\
(0.01)\end{array}$ & $\begin{array}{l}{[0.01} \\
0.07]\end{array}$ & $\begin{array}{l}0.04^{*} \\
(0.02)\end{array}$ & $\begin{array}{l}{[0.01} \\
0.07]\end{array}$ \\
\hline NSE quintil 4 & $\begin{array}{l}0.02 \\
(0.01)\end{array}$ & $\begin{array}{l}{[0,} \\
0.04]\end{array}$ & $\begin{array}{l}0.03 \\
(0.01)\end{array}$ & $\begin{array}{l}{[0,} \\
0.05]\end{array}$ & $\begin{array}{l}0.04^{*} \\
(0.01)\end{array}$ & $\begin{array}{l}{[0.01} \\
0.07]\end{array}$ & $\begin{array}{l}0.04 * \\
(0.02)\end{array}$ & $\begin{array}{l}{[0.01} \\
0.07]\end{array}$ \\
\hline NSE quintil 5 & $\begin{array}{l}0.01 \\
(0.01)\end{array}$ & $\begin{array}{l}{[-0.02} \\
0.03]\end{array}$ & $\begin{array}{l}0.01 \\
(0.02)\end{array}$ & $\begin{array}{l}{[-0.02} \\
0.05]\end{array}$ & $\begin{array}{l}0.04 \\
(0.02)\end{array}$ & $\begin{array}{l}{[0.00} \\
0.07]\end{array}$ & $\begin{array}{l}0.03 \\
(0.02)\end{array}$ & $\begin{array}{l}{[-0.01} \\
0.07]\end{array}$ \\
\hline $\begin{array}{l}\text { Interés de padres } \\
\text { en política }\end{array}$ & & & $\begin{array}{l}-0.01 \\
(0.00)\end{array}$ & $\begin{array}{l}{[-0.01} \\
0.00]\end{array}$ & & & $\begin{array}{l}0.00 \\
(0.00)\end{array}$ & $\begin{array}{l}{[-0.01,} \\
0.01]\end{array}$ \\
\hline $\begin{array}{l}\text { Discutir sobre } \\
\text { política fuera de } \\
\text { la escuela }\end{array}$ & & & $\begin{array}{l}0.01 \\
(0.00)\end{array}$ & $\begin{array}{l}{[0,00} \\
0.01]\end{array}$ & & & $\begin{array}{l}0.00 \\
(0.01)\end{array}$ & $\begin{array}{l}{[-0.01} \\
0.01]\end{array}$ \\
\hline $\begin{array}{l}\text { Dependencia } \\
\text { (privado) }\end{array}$ & & & $\begin{array}{l}-0.01 \\
(0.01)\end{array}$ & $\begin{array}{l}{[-0.03} \\
0.01]\end{array}$ & & & $\begin{array}{l}0.01 \\
(0.01)\end{array}$ & $\begin{array}{l}{[-0.02,} \\
0.03]\end{array}$ \\
\hline NSE Escuela & & & $\begin{array}{l}0.00 \\
(0.01)\end{array}$ & $\begin{array}{l}{[-0.01} \\
0.01]\end{array}$ & & & $\begin{array}{l}0.00 \\
(0.01)\end{array}$ & $\begin{array}{l}{[-0.02,} \\
0.01]\end{array}$ \\
\hline $\begin{array}{l}\text { Discusión abierta } \\
\text { en clases }\end{array}$ & & & $0(0)$ & $\begin{array}{l}{[-0.01} \\
0.01]\end{array}$ & & & $\begin{array}{l}0.01 \\
(0.00)\end{array}$ & $\begin{array}{l}{[0,00} \\
0.02]\end{array}$ \\
\hline ICC & 0.01 & & 0.01 & & 0.02 & & 0.02 & \\
\hline
\end{tabular}

Nota: * $p<.05$; LI y LS son los límites inferior y superior de los intervalos al 95\% de confianza. Fuente: elaboración propia.

Finalmente, la Tabla 7 despliega los resultados de las estimaciones de conocimiento cívico ${ }^{7}$, pudiéndose destacar tres aspectos. En primer lugar, se puede observar que en ambos años el género es estadísticamente significativo en todos los modelos, siempre a favor de las mujeres. Además, hay un crecimiento de este efecto en el tiempo, ya que mientras en 2009 las mujeres tenían una ventaja promedio de 0.09 desviaciones estándar respecto a los hombres (controlando por todas las variables del modelo), en 2016, dicha diferencia era de 0.22 desviaciones estándar. Esto podría estar indicando una temprana diferencia a favor de las mujeres respecto de la capacidad de

7 Para el modelamiento multinivel el indicador de conocimiento cívico se estandarizó (media 0 y desviación estándar 1), de manera de hacer comparables las magnitudes de los coeficientes entre años. 
comprender los problemas sociales y entender el entorno político y cívico que les rodea, tendencia que es internacional y no se limita solo al conocimiento cívico (Sandoval-Hernández, Isac y Miranda, 2018).

En segundo lugar, se observa que el conocimiento cívico está fuertemente influenciado por el nivel socioeconómico promedio de la escuela y del estudiante. Así, cuanto mayor es el nivel socioeconómico mayor es el conocimiento cívico, aunque, a diferencia de lo que ocurre con el género, la diferencia en el nivel socioeconómico se mantiene estable entre 2009 y 2016. Finalmente, otras variables ya relevadas por estudios anteriores, como el rol de la discusión en la sala de clases y fuera de la escuela (Carrasco, Banerjee, Treviño y Villalobos, 2020; Treviño et al., 2017) son estadísticamente significativos en ambos años, aunque con magnitudes similares.

Tabla 7. Modelo multinivel. Conocimiento Cívico

\begin{tabular}{|c|c|c|c|c|c|c|c|c|}
\hline & \multicolumn{4}{|c|}{2009} & \multicolumn{4}{|c|}{2016} \\
\hline & \multicolumn{2}{|c|}{ Modelo 1} & \multicolumn{2}{|c|}{ Modelo 2} & \multicolumn{2}{|c|}{ Modelo 3} & \multicolumn{2}{|c|}{ Modelo 4} \\
\hline & $\begin{array}{l}\text { Coef } \\
\text { (se) }\end{array}$ & LI, LS & $\begin{array}{l}\text { Coef } \\
\text { (se) }\end{array}$ & LI, LS & $\begin{array}{l}\text { Coef } \\
\text { (se) }\end{array}$ & LI, LS & $\begin{array}{l}\text { Coef } \\
\text { (se) }\end{array}$ & LI, LS \\
\hline Intercepto & $\begin{array}{l}-0.66 \\
(0.07)\end{array}$ & $\begin{array}{l}{[-0.8} \\
-0.52]\end{array}$ & $\begin{array}{l}-0.19 \\
(0.06)\end{array}$ & $\begin{array}{l}{[-0.31} \\
-0.07]\end{array}$ & $\begin{array}{l}-0.69 * \\
(0.05)\end{array}$ & $\begin{array}{l}{[-0.79} \\
-0.59]\end{array}$ & $\begin{array}{l}-0.32^{*} \\
(0.06)\end{array}$ & $\begin{array}{c}{[-0.44,} \\
-0.2]\end{array}$ \\
\hline $\begin{array}{l}\text { Género (Feme- } \\
\text { nino) }\end{array}$ & $\begin{array}{l}0.16^{*} \\
(0.04)\end{array}$ & $\begin{array}{l}{[0.07} \\
0.25]\end{array}$ & $\begin{array}{l}0.09 * \\
(0.03)\end{array}$ & $\begin{array}{l}{[0.03} \\
0.16]\end{array}$ & $\begin{array}{l}0.24^{*} \\
(0.04)\end{array}$ & $\begin{array}{l}{[0.18} \\
0.31]\end{array}$ & $\begin{array}{l}0.22^{*} \\
(0.03)\end{array}$ & $\begin{array}{r}{[0.15} \\
0.29]\end{array}$ \\
\hline NSE quintil 2 & $\begin{array}{l}0.22^{*} \\
(0.07)\end{array}$ & $\begin{array}{l}{[0.08} \\
0.36]\end{array}$ & $\begin{array}{c}0.03 \\
(0.06)\end{array}$ & $\begin{array}{r}{[-0.09} \\
0.14]\end{array}$ & $\begin{array}{c}0.3^{*} \\
(0.05)\end{array}$ & $\begin{array}{c}{[0.2} \\
0.4]\end{array}$ & $\begin{array}{c}0.08 \\
(0.05)\end{array}$ & $\begin{array}{c}{[-0.02} \\
0.18]\end{array}$ \\
\hline NSE quintil 3 & $\begin{array}{l}0.46^{*} \\
(0.07)\end{array}$ & $\begin{array}{c}{[0.33} \\
0.6]\end{array}$ & $\begin{array}{l}0.16^{*} \\
(0.06)\end{array}$ & $\begin{array}{r}{[0.05} \\
0.26]\end{array}$ & $\begin{array}{c}0.5^{*} \\
(0.05)\end{array}$ & $\begin{array}{l}{[0.4} \\
0.61]\end{array}$ & $\begin{array}{l}0.19^{*} \\
(0.05)\end{array}$ & $\begin{array}{c}{[0.09,} \\
0.3]\end{array}$ \\
\hline NSE quintil 4 & $\begin{array}{l}0.76^{*} \\
(0.07)\end{array}$ & $\begin{array}{c}{[0.63} \\
0.9]\end{array}$ & $\begin{array}{l}0.28^{*} \\
(0.05)\end{array}$ & $\begin{array}{l}{[0.18} \\
0.39]\end{array}$ & $\begin{array}{l}0.78^{*} \\
(0.06)\end{array}$ & $\begin{array}{c}{[0.67} \\
0.9]\end{array}$ & $\begin{array}{l}0.28^{*} \\
(0.06)\end{array}$ & $\begin{array}{c}{[0.16,} \\
0.4]\end{array}$ \\
\hline NSE quintil 5 & $\begin{array}{l}1.18^{*} \\
(0.08)\end{array}$ & $\begin{array}{r}{[1.03,} \\
1.34]\end{array}$ & $\begin{array}{l}0.43^{*} \\
(0.06)\end{array}$ & $\begin{array}{l}{[0.31} \\
0.55]\end{array}$ & $\begin{array}{l}1.12^{*} \\
(0.06)\end{array}$ & $\begin{array}{c}{[1} \\
1.24]\end{array}$ & $\begin{array}{l}0.35^{*} \\
(0.07)\end{array}$ & $\begin{array}{r}{[0.21} \\
0.48]\end{array}$ \\
\hline $\begin{array}{l}\text { Interés de padres } \\
\text { en política }\end{array}$ & & & $\begin{array}{c}0.01 \\
(0.01)\end{array}$ & $\begin{array}{c}{[-0.02} \\
0.04]\end{array}$ & & & $\begin{array}{c}0.03 \\
(0.02)\end{array}$ & $\begin{array}{c}{[-0.01} \\
0.06]\end{array}$ \\
\hline $\begin{array}{l}\text { Discutir sobre } \\
\text { política fuera de } \\
\text { la escuela }\end{array}$ & & & $\begin{array}{l}0.07 * \\
(0.02)\end{array}$ & $\begin{array}{c}{[0.04} \\
0.1]\end{array}$ & & & $\begin{array}{l}0.06^{*} \\
(0.01)\end{array}$ & $\begin{array}{r}{[0.03} \\
0.09]\end{array}$ \\
\hline $\begin{array}{l}\text { Dependencia } \\
\text { (privado) }\end{array}$ & & & $\begin{array}{l}-0.07 \\
(0.06)\end{array}$ & $\begin{array}{c}{[-0.18} \\
0.05]\end{array}$ & & & $\begin{array}{c}0.08 \\
(0.06)\end{array}$ & $\begin{array}{c}{[-0.05,} \\
0.2]\end{array}$ \\
\hline NSE Escuela & & & $\begin{array}{l}0.36^{*} \\
(0.03)\end{array}$ & $\begin{array}{l}{[0.3} \\
0.42]\end{array}$ & & & $\begin{array}{l}0.34^{*} \\
(0.04)\end{array}$ & $\begin{array}{r}{[0.27} \\
0.42]\end{array}$ \\
\hline $\begin{array}{l}\text { Discusión abierta } \\
\text { en clases }\end{array}$ & & & $\begin{array}{l}0.16^{*} \\
(0.03)\end{array}$ & $\begin{array}{l}{[0.1} \\
0.22]\end{array}$ & & & $\begin{array}{l}0.14^{*} \\
(0.03)\end{array}$ & $\begin{array}{r}{[0.09,} \\
0.19]\end{array}$ \\
\hline ICC & 0.28 & & 0.28 & & 0.29 & & 0.28 & \\
\hline
\end{tabular}

Nota: ${ }^{*} p<.05$; LI y LS son los límites inferior y superior de los intervalos al $95 \%$ de confianza. Fuente: elaboración propia 


\section{Conclusiones}

Las ILSA han sido motivo de un creciente debate académico durante la última década. Aunque poco tematizado en este debate, el ICCS permite observar cómo un ILSA puede informar sobre un aspecto integral de la educación como es la ciudadanía. Considerando esto, el estudio analizó las tendencias de cambio en cuatro variables relacionadas con la educación cívica y ciudadana en Chile (conocimiento cívico, disposición a votar en las elecciones, confianza en los partidos políticos y disposición a compartir con otros de distinta clase social) entre 2009 y 2016, considerando los cambios en programas y políticas buscaron transformar algunos de estos elementos durante este tiempo.

Aunque los resultados generales mostraron pocos cambios entre los jóvenes al comparar entre años, el análisis por nivel socioeconómico y el análisis multinivel evidenciaron que algunos cambios (p.ej., el cambio en la disposición a compartir con otros o en la disposición a votar en las elecciones) se producían de manera diferenciada entre grupos sociales; o con cambios relevantes en la magnitud de otras variables, como en el caso del género; aunque otras variables (p.e., la discusión en la sala de clases) se mostraron estables entre 2009 y 2016 , entregando un panorama heterogéneo de las tendencias en el tiempo.

Aunque particularizados en un país, este conjunto de resultados permite re-discutir la relación entre las ILSA y las políticas educativas, pudiendo destacarse tres aspectos. En primer lugar, es interesante observar que, aún en contextos de implementación de políticas y programas enfocados a promover transformaciones, los resultados del ICCS entre 2009 y 2016 muestran gran estabilidad. Esto sugiere que los resultados de ciudadanía no parecen haber "permeado" las discusiones sobre políticas -como si ocurriría, por ejemplo, en TIMSS o PISA (Parcerisa y Verger, 2019)-, ya que no se evidencian cambios que hayan permitido formar estudiantes con mayores niveles de conocimiento cívico, más dispuestos a compartir con otros y a participar de manera más activa en los procesos de democracia participativa. Evidentemente, esta estabilidad puede deberse a múltiples factores -como la baja capacidad transformativa de las políticas, el peso de los factores culturales o la potencia del mercado educativo del país, entre otras- pero es interesante mostrar la baja capacidad de cambio que ha mostrado el sistema, aún en posesión de evidencia de resultados poco alentadores para la formación ciudadana de los estudiantes.

En segundo término, esta estabilidad contrasta con los cambios evidenciados cuando se desagrega por nivel socioeconómico. Esto permite discutir ya no tanto la existencia misma de la información, sino su proceso de presentación y difusión. El énfasis entregado a los promedios y el pro- 
cesamiento mediático que se hace a los datos de las ILSA (Pizmony-Levy y Torney-Purta, 2018 ) reduce la riqueza de los datos, no permitiendo entender los alcances de las políticas ni tampoco entendiendo cómo estas políticas afectan de manera diferenciada a los estudiantes según las distintas posiciones en la estructura social que estos ocupan. Asimismo, estos análisis generales tampoco permiten ver efectos que aumentan la desigualdad, como el aumento en las brechas a la disposición a votar evidenciadas en Chile entre 2009 y 2016 . Por este motivo, se requieren análisis más sofisticados que den cuenta del comportamiento de las relaciones a lo largo de la distribución de la variable de interés.

Finalmente, y algo que se puede colegir a partir de la interpretación de los resultados de los modelos multinivel, es interesante pensar en cómo los datos de las ILSA pueden orientar no sólo políticas dentro de la estructura del sistema educativo, sino también iluminar otros aspectos de las sociedades. Esto refiere, en último término, a una discusión sobre el uso que se hace de estas evaluaciones. Así, creemos que es posible pensar y promover que las ILSA no sean usados como "barómetros de calidad" de los sistemas educativos (Carnoy et al., 2013), que generen una competencia entre sistemas y que orienten cambios radicales siguiendo tendencias extranjeras (Parcerisa et al., 2020; Simola, 2013) sino utilizar los datos para orientar cambios en políticas sociales, educativas, económicas que permitan mostrar espacios de mejora de los estudiantes, sin "culpar" inmediatamente a los sistemas educativos por estos resultados. Así, por ejemplo, en el caso chileno, los resultados analizados sobre voto, disposición a compartir con otros y confianza en los partidos políticos parecen ser un reflejo de problemas estructurales de la sociedad, que no se limitan necesariamente a cambios particulares del sistema educativo, sino que requieren transformaciones en los cimientos (constitucionales) del país.

\section{Agradecimientos}

El artículo se desarrolla en el marco del proyecto Fondecyt Regular $N^{\circ} 1180667$. Adicionalmente, los autores agradecen el apoyo brindado por el proyecto CONICYT PIA CIE160007. Ambos proyectos son financiados por Agencia Nacional de Investigación y Desarrollo (ANID) de Chile. Finalmente, los autores agradecen los comentarios y sugerencias de Diego Carrasco y Lluís Parcerisa, así como los comentarios y sugerencias de los/as revisores/ as anónimos. 


\section{Referencias}

Alarcón, C. (2020). An Improbable Identification? The Netherlands as a "Reference Society" within the Chilean Educational Policy-Making Debate (2014-2015). European Education, 52(1), 48-67. https://doi.org/10.1080/10564934.2019.1694418

Alvarado, E., Rivera, P., y Morales, R. (2019). Radicalizing democracy from social movements. The compared cases of Podemos in Spain and Frente Amplio in Chile. Izquierdas, 48, 87-105. https://doi.org/10.4067/S0718-50492019000400087

Ball, S. J. (2003). The teacher's soul and the terrors of performativity. Journal of Education Policy, 18(2), 215-228. https://doi.org/10.1080/0268093022000043065

Bieber, T. (2016). Soft governance, international organizations and education policy convergence: comparing PISA and the Bologna and Copenhagen processes. Amsterdam: Springer.

Canales, M., Bellei, C., y Orellana, V. (2016). ¿Por qué elegir una escuela privada subvencionada? Sectores medios emergentes y elección de escuela en un sistema de mercado. Estudios Pedagogicos, 42(3), 89-109. https://doi.org/10.4067/s0718-07052016000400005

Cariola, L., Covacevich, C., Gubler, J., Lagos, E., y Ortiz, M. (2011). Chilean participation in IEA Studies. In C. Papanastasiou, T. Plomp, y E. Papanastasiou (Eds.), IEA 1958-2008: 50 Years of Experiences and Memories. Nicosia: Cultural Center of the Kykkos Monastery (pp. 373-387).

Carlin, R. E. (2006). The decline of citizen participation in electoral politics in post-authoritarian Chile. Democratization, 13(4), 632-651. https://doi.org/10.1080/13510340600791921

Carnoy, M., Khavenson, T., y Ivanova, A. (2013). Using TIMSS and PISA results to inform educational policy: a study of Russia and its neighbours. Compare, 45(2), 248-271. https://doi.org/10.1080/03057925.2013.855002

Carrasco, D., Banerjee, R., Treviño, E., y Villalobos, C. (2020). Civic knowledge and open classroom discussion: explaining tolerance of corruption among 8th-grade students in Latin America. Educational Psychology, 40(2), 186-206. https://doi.org/10.1080/0144 $\underline{3410.2019 .1699907}$

Corvalán, A., y Cox, P. (2013). Class-Biased Electoral Participation: The Youth Vote in Chile. Latin American Politics and Society, 55(03), 47-68. https://doi.org/10.1111/j.15482456.2013.00202.x

Cox, C. (en prensa). IEA Civic Education Studies in Latin America: paths of influence and critique in policy and research. In J Torney-Purta y B. Malak-Minkiewicz (Eds.), Civic and Citizenship Education Studies of IEA: Influences on Practice, Policy and Research. Amsterdam: IEA.

Cox, C., y García, C. (2017). Evolution of citizenship education in Chile: Recent curricula compared. In García-Cabrero, B., Sandoval-Hernández, A., Treviño, E., Diazgrandos-Ferrand, S. y Perez, G. (Eds.). Civics and Citizenship. Theoretical Models and Experiences in Latin America. Rotterdam/Boston/Taipei: Sense Publisher (pp. 85-103).

Cox, C., y Meckes, L. (2016). International large-scale assessment studies and educational policy-making in Chile: contexts and dimensions of influence. Research Papers in Education, 31(5), 502-515. https://doi.org/10.1080/02671522.2016.1225349

Gorur, R. (2017). Towards productive critique of large-scale comparisons in education. Critical Studies in Education, 58(3), 341-355. https://doi.org/10.1080/17508487.2017 .1327876 
Grek, S. (2009). Governing by numbers: The PISA "effect" in Europe. Journal of Education Policy, 24(1), 23-37. https://doi.org/10.1080/02680930802412669

Heyneman, S., y Lee, B. (2014). The impact of international studies of academic achievement on policy and research. In Rutkowski, L., von Davier, M. y Rutkowski, R. (Eds.). Handbook of International Large-Scale Assessment: Background, technical issues and methods of data analysis. New York: CRC Press (pp. 37-72).

Hox, J., Moerbeek, M., y Van de Schoot, R. (2017). Multilevel analysis: Techniques and applications. Routledge.

INJUV (2009). Sexta encuesta nacional de juventud. Santiago, Chile.

Isac, M. M., Maslowski, R., Creemers, B., y van der Werf, G. (2014). The contribution of schooling to secondary-school students' citizenship outcomes across countries. School Effectiveness and School Improvement, 25(1), 29-63. https://doi.org/10.1 $\underline{080 / 09243453.2012 .751035}$

Klemencic, E. (2010). The impact of international achievement studies on national education policymaking: The case of Slovenia - how many watches do we need? International Perspectives on Education and Society, 13, 239-266. https://doi.org/10.1108/ S1479-3679(2010)0000013012

Knowles, R. T., Torney-Purta, J., y Barber, C. (2018). Enhancing citizenship learning with international comparative research: Analyses of IEA civic education datasets. Citizenship Teaching and Learning, 13(1), 7-30. https://doi.org/10.1386/ctl.13.1.7_1

MINEDUC. (2013). Bases Curriculares 2013. Historia, Geografía y Ciencias Sociales. Santiago, Chile.

Niemann, D., y Martens, K. (2018). Soft governance by hard fact? The OECD as a knowledge broker in education policy. Global Social Policy, 18(3), 267-283. https://doi. org/10.1177/1468018118794076

Ozga, J. (2008). Governing Knowledge: Research Steering and Research Quality. European Educational Research Journal, 7(3), 261-272. https://doi.org/10.2304/ eerj.2008.7.3.261

Parcerisa, L., y Falabella, A. (2017). La consolidación del estado evaluador a través de políticas de rendición de cuentas: trayectoria, producción y tensiones en el sistema educativo chileno. Education Policy Analysis Archives/Archivos Analíticos de Políticas Educativas, 25, 1-24. https://doi.org/10.14507/epaa.25.3177

Parcerisa, L., Fontdevila, C., y Verger, A. (2020). Understanding the PISA influence on national education policies: a focus on policy transfer mechanisms. In S. Jornitz y A. Wilmers (Eds.), International perspectives on school settings, education policy and digital strategies. A transatlantic discourse in education research. Lerverkusen.

Parcerisa, L., y Verger, A. (2019). PISA projections in Chile: The selective use of league leaders in the enactment of recent education reforms. In Wladow, F. y Steiner-Khamsi, G. (Eds.). Understanding PISA's attractiveness: Critical analyses in comparative policy studies. Bloomsbury Academic (pp. 25-48).

Pelgrum, H., Blahova, V., Dukynait , R., Paveši , B. J., y Kangro, A. (2011). IEA experiences from Latvia, Lithuania, Slovak Republic and Slovenia. In C. Papanastasiou, T. Plomp, y E. Papanastasiou (Eds.), IEA 1958-2008: 50 Years of Experiences and Memories. Nicosia: Cultural Center of the Kykkos Monastery (pp. 447-468).

Pizmony-Levy, O., y Torney-Purta, J. (2018). How journalists and researchers communicate results of international large-scale assessments. Cadmo, 26(1), 51-65. https://doi. org/10.3280/CAD2018-001007 
Rioux, C., y Little, T. D. (2020). Underused Methods in Developmental Science to Inform Policy and Practice. Child Development Perspectives, 14(2), 97-103. https://doi. org/10.1111/cdep.12364

Sandoval-Hernández, A., Isac, M., y Miranda, D. (Eds.) (2018). Teaching tolerance in a globalized world. Amsterdam: Springer-IEA.

Schulz, W., Fraillon, J., Ainley, J., Losito, B., y Kerr, D. (2008). International Civic and Citizenship Education Study: Assessment Framework. Amsterdam: International Association for the Evaluation of Educational Achievement (IEA).

Sellar, S., Lingard, B., Rutkowski, D., y Takayama, K. (2018). Student preparation for large-scale assessments: a comparative analysis. In Madoxx, B. (Ed.). International Large-Scale Assessments in Education. Insider Research Perspective. London, UK: Bloomsbury. (pp. 137-155).

Simola, H. (2013). El milagro finlandés de PISA: observaciones históricas y sociológicas sobre la enseñanza y la formación del profesorado. Revista de Currículum y Formación Del Profesorado, 17(2), 153-169.

Torney-Purta, J., y Amadeo, J.-A. (2013). International Large-Scale Assessments: Challenges in Reporting and Potentials for Secondary Analysis. Research in Comparative and International Education, 8(3), 248-258. https://doi.org/10.2304/rcie.2013.8.3.248

Treviño, E., Béjares, C., Villalobos, C., y Naranjo, E. (2017). Influence of teachers and schools on students' civic outcomes in Latin America. The Journal of Educational Research, 110(6), 604-618. https://doi.org/10.1080/00220671.2016.1164114

Treviño, E., Villalobos, C., Béjares, C., y Naranjo, E. (2019). Forms of youth political participation and educational system: The role of the school for 8 th grade students in Chile. Young, 27(3), 279-303. https://doi.org/10.1177/1103308818787691

Valenzuela, J. P., Bellei, C., y De los Ríos, D. (2014). Socioeconomic school segregation in a market-oriented educational system. The case of Chile. Journal of Education Policy, 29(2), 217-241. https://doi.org/10.1080/02680939.2013.806995

Valenzuela, J. P., Villarroel, G., y Villalobos, C. (2013). Ley de Subvención Escolar Preferencial (SEP): algunos resultados preliminares de su implementación. Revista Pensamiento Educativo, 50(2), 113-131. https://doi:10.7764/PEL.50.2.2013.7

Villalobos, C., y Quaresma, M. L. (2015). Sistema escolar chileno: Características y consecuencias de un modelo orientado al mercado. Convergencia, 22(69), 63-84. https://doi. org/10.29101/crcs.v22i69.3634

Villalobos, C., y Valenzuela, J. P. (2012). Polarización y cohesión social del sistema escolar chileno. Revista de Análisis Económico, 27(2), 145-172. http://dx.doi.org/10.4067/ $\underline{\text { S0718-88702012000200005 }}$

Wagemaker, H. (2011). IEA: International studies, impact and transition. In C. Papanastasiou, T. Plomp, y E. Papanastasiou (Eds.), IEA 1958-2008: 50 years of experiences and memories. Nicosia: Cultural Center of the Kykkos Monastery (pp. 257-272).

Wagemaker, H. (2013). International large-scale assessments: From research to policy. In Rutkowski, L., von Davier, M. y Rutkowski, R. (Eds.). Handbook of international large-scale assessment: Background, technical issues, and methods of data analysis. New York: CRC Press (pp. 11-36).

Weinstein, J., y Villalobos, C. (2016). Chile: la experiencia de la Subvención Escolar Preferencial. In Souto, M. (Ed.). Transferencias directas a escuelas: reflexiones sobre prácticas en América Latina. IPPE: Buenos Aires (pp. 58-100). 